\title{
On the folding and deployment of tape springs: A large displacements and large rotations rod model with highly flexible thin-walled cross-section.
}

\author{
E. Picault* \\ Laboratoire de Mécanique et d'Acoustique, CNRS UPR 7051, Marseille, France. \\ S. Bourgeois ${ }^{\dagger}$ and B. Cochelin ${ }^{\ddagger}$ \\ Laboratoire de Mécanique et d'Acoustique, CNRS UPR 7051, Marseille, France. \\ Ecole Centrale Marseille, Marseille, France. \\ F. Guinot ${ }^{\S}$ \\ Thales Alenia Space, Cannes La Bocca, France.
}

In the framework of deployable structures, we focus on the modeling of tape springs, i.e. rod-like elastic bodies with thin-walled cross-section which develop localized folds due to a flattening of the cross-section. A rod model with highly deformable cross-section and few kinematics parameters is derived from a complete shell model, for large displacements, large rotations and dynamics. The simplicity of the model is achieved by introducing an elastica kinematics to describe the changes in the cross-section shape. This model is able to handle the formation of localized folds which can move along the rod line, merge or split, allowing to simulate complex scenarios of folding and deployment.

\section{Nomenclature}

$h$

$L$

$s$

$s$

$t$

$O$

G

$G$

$\mathrm{e}$

e

a

$y \quad$ First coordinate in the cross-section plane $(\mathrm{m})$

$z \quad$ Second coordinate in the cross-section plane $(\mathrm{m})$ $\begin{array}{ll}u_{1} & \text { Translation of the cross-section along } \mathbf{e}_{\mathbf{1}}(\mathrm{m}) \\ u_{3} & \text { Translation of the cross-section along } \mathbf{e}_{\mathbf{3}}(\mathrm{m}) \\ \theta & \text { Rotation of the cross-section around } \mathbf{e}_{\mathbf{2}}(\mathrm{rad}) \\ \beta & \text { Opening angle of the cross-section curve (rad) } \\ \beta^{e} & \text { Opening angle for } s_{2}=a / 2(\mathrm{rad}) \\ e_{i j} & \text { Membrane strains } \\ k_{i j} & \text { Bending strains }\left(\mathrm{m}^{-1}\right) \\ U_{e} & \text { Strain energy }(\mathrm{J}) \\ T & \text { Kinetic energy }(\mathrm{J}) \\ A & \text { Tensile stiffness of the shell }(\mathrm{N}) \\ D_{k} & \text { Bending stiffnesses of the shell }\left(\mathrm{N} . \mathrm{m}^{2}\right) \\ \rho & \left.\text { Density (kg.m }{ }^{-3}\right) \\ E & \text { Young's modulus }(\mathrm{Pa}) \\ \nu & \text { Poisson's ratio }\end{array}$

\footnotetext{
*PhD Student, Laboratoire de Mécanique et d'Acoustique, picault@lma.cnrs-mrs.fr.

$\dagger$ Associate Professor, Ecole Centrale Marseille, stephane.bourgeois@centrale-marseille.fr.

$\ddagger$ Professor, Ecole Centrale Marseille, bruno.cochelin@centrale-marseille.fr.

$\S$ Research Engineer, Thales Alenia Space, francois.guinot@thalesaleniaspace.com.
} 


\section{Introduction}

A major challenge for satellite manufacturers is to develop ingenious systems as compact as possible in order to optimize the volume needed for transportation and storage. Deployable structures are one of the means used to answer these problems. In particular, deployable elastic structures are an interesting alternative to articulated rigid structures with hinges and bolts, thanks to their high potential of flexibility. Indeed, they offer better compacity through folding or coiling and are capable of deploying themselves in an autonomous way during the set-up.

Tape springs are an example of such slender elastic structures. This kind of structures has been widely studied in the nonlinear framework ${ }^{2,9,16}$ since they suffer from geometrical instabilities that can lead to a sudden loss of stiffness as well as extreme deformation shapes, as shown in Figure 1. Moreover their various applications in the aerospace field ${ }^{11,12}$, but also in biophysics, biomechanics or even in micro or nanomechanics ${ }^{15}$ are as many motivations to develop robust models ${ }^{1,14}$.

In its free state, a tape spring can be assimilated to a straight thin-walled beam with a circular open cross-section of constant transverse curvature. Under progressively applied bending or compressive loads (see Figure 1), this structure will behave at first like a beam before the sudden appearance of localized folds. These folds indicate snap-through buckling ${ }^{12}$ and appear thanks to a localized flattening of the cross-section that drastically reduces the moment of inertia, leading to the concentration of the bending deformation in the fold area. Away from the fold, the tape spring remains almost straight and undeformed. Thus the deformed tape spring may be divided into three characteristic areas: the folding area, an undeformed area and a transitional area. Everyone can experience with a Carpenter tape the formation of one or several folds, their motion along the tape, as well as the splitting of a single fold into two or the merging of two folds into one...

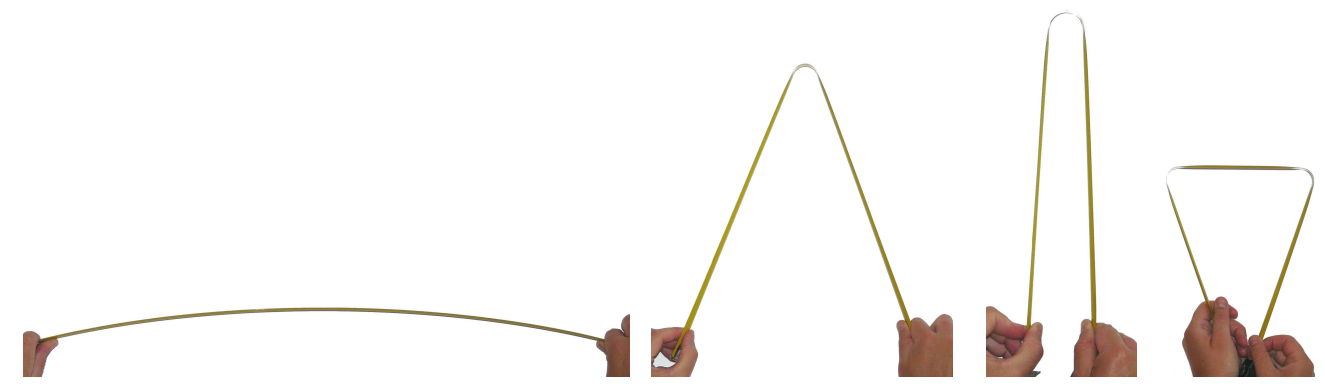

Figure 1. Folding of a tape spring.

The modeling of tape springs has already been addressed in literature and can be classified into two main approaches. The first one consists in the full computation of a nonlinear shell model for large displacements and large rotations, and produces precise results in static and dynamic for any loading configuration and boundary conditions ${ }^{13,18,8}$. But this approach does not take into account the specificity of a tape spring and leads to hard to drive calculations and heavy simulations.

The second approach consists in the use of a combination of rigid bars and nonlinear spiral springs to model the tape and was inspired by the observation that a tape spring remains straight and undeformed away from the folds ${ }^{12}$. Thus the spiral springs account for the stiffness of the fold areas, and the rigid bars of variable lengths represent the undeformed parts. This kind of model allows the simulation of complex deployment scenarios with very few parameters but requires the introduction of the folds ab initio, which limits the panel of scenarios that can be simulated.

An intermediate approach would consist in modeling tape springs as rods with highly deformable crosssection. Several methods are used to account for the deformation of the cross-section in classical beam models, e.g. the introduction of additional degrees of freedom ${ }^{17,4,10}$ or the discretization of the crosssection with finite elements ${ }^{19}$. The model proposed herein is based on this intermediate approach but takes 
into account the possibility of large relative displacements in the cross-section with a kinematics more suited for tape springs.

A one-dimensional continuous rod-like model is derived from a nonlinear shell model for large displacements, large rotations and dynamics thanks to the introduction of kinematic and sthenic assumptions on the behavior of the cross-section. In addition to the classical hypotheses of beam theory, the model's originality relies on the use of an elastica kinematics to describe the cross-section deformed shape with few parameters. These assumptions are used to reduce the expressions of the shell energies before performing an analytical integration over the cross-section in order to obtain the one-dimensional model. Using the Hamilton's principle, these expressions are then directly introduced in the FE software COMSOL which performs an automatic differentiation. The obtained model is appropriate for simulating complex folding and deployment scenarios ${ }^{6,7}$.

\section{The rod model}

\section{II.A. Kinematic description and basic assumptions}

We consider a tape spring regarded as a rod, and made of a planar circular cross-section curve extruded along an initially straight rod line as shown in Figure 2. A fixed orthonormal frame $\left(O, \mathbf{e}_{\mathbf{1}}, \mathbf{e}_{\mathbf{2}}, \mathbf{e}_{\mathbf{3}}\right)$ is chosen such that the axis $\left(O, \mathbf{e}_{\mathbf{1}}\right)$ is directed along the rod line and that the plane $\left(O, \mathbf{e}_{\mathbf{1}}, \mathbf{e}_{\mathbf{3}}\right)$ is the plane of symmetry of the cross-section curve.
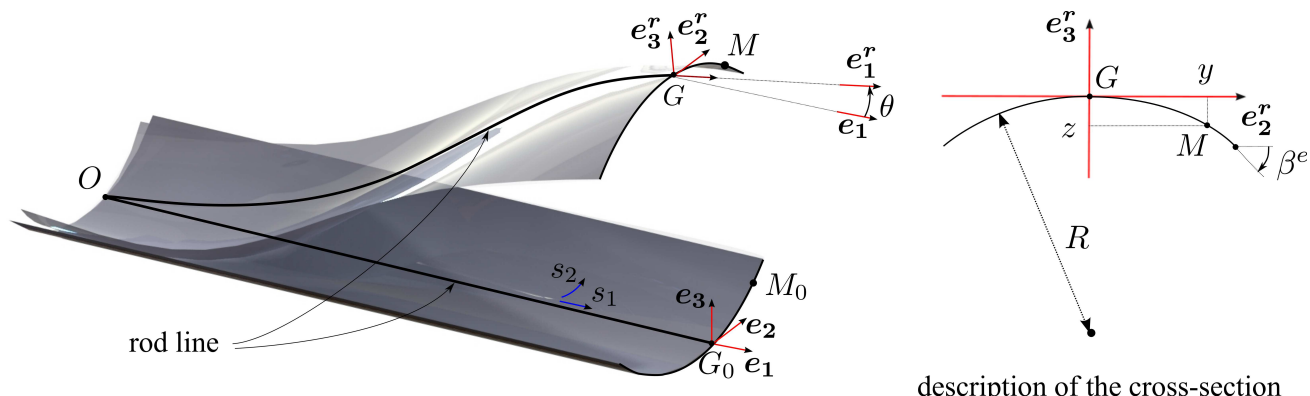

description of the cross-section

Figure 2. Parametrization of a tape spring.

A curvilinear coordinate system $\left(s_{1}, s_{2}\right) \in[0, L] \times[-a / 2, a / 2]$ is then introduced to describe the geometry of the tape spring, with $L$ the initial length of the rod line and $a$ the initial length of the cross-section curve. In the deformed configuration, the position of a material point $M$ is given by:

$$
\mathbf{O M}\left(s_{1}, s_{2}, t\right)=\mathbf{O G}\left(s_{1}, t\right)+\mathbf{G M}\left(s_{1}, s_{2}, t\right)
$$

where $G$ is the point of the cross-section attached to the rod line.

Given the load cases we will consider, we restrict our study to the plane case. Thus the motion of the rod line is restrained to the plane $\left(O, \mathbf{e}_{\mathbf{1}}, \mathbf{e}_{\mathbf{3}}\right)$ whereas the cross-section curve, initially symmetric with respect to this plane, is assumed to remain symmetric in any deformed configuration. The cross-section position is then given by:

$$
\mathbf{O G}\left(s_{1}, t\right)=\left[s_{1}+u_{1}\left(s_{1}, t\right)\right] \mathbf{e}_{\mathbf{1}}+u_{3}\left(s_{1}, t\right) \mathbf{e}_{\mathbf{3}}
$$

where $u_{1}\left(s_{1}, t\right)$ and $u_{3}\left(s_{1}, t\right)$ are the translations of the material point $G$.

The proposed rod model kinematics relies on three assumptions:

(i) the cross-section curve remains in a plane after deformation,

(ii) the section plane is orthogonal to the tangent vector of the rod line in the deformed configuration,

(iii) the cross-section curve is considered inextensible. 
The two first assumptions are classical hypotheses of Euler-Bernoulli beam theory. Assumption (i) makes it possible to introduce a rotated frame $\left(G, \mathbf{e}_{1}^{\mathbf{r}}, \mathbf{e}_{2}^{\mathbf{r}}, \mathbf{e}_{3}^{\mathbf{r}}\right)$, image of the frame $\left(G, \mathbf{e}_{1}, \mathbf{e}_{2}, \mathbf{e}_{3}\right)$ through the rotation of angle $\theta\left(s_{1}, t\right)$ around the axis $\mathbf{e}_{2}$ : $\mathbf{e}_{1}^{\mathbf{r}}$ is a unit vector orthogonal to the section plane in the deformed configuration, $\mathbf{e}_{2}^{\mathbf{r}}$ is set to $\mathbf{e}_{2}$ and $\mathbf{e}_{3}^{\mathbf{r}}=\mathbf{e}_{1}^{\mathbf{r}} \times \mathbf{e}_{2}^{\mathbf{r}}$. Assumption (ii) implies that the vector $\mathbf{e}_{1}^{\mathbf{r}}$ is collinear to the natural rod line tangent vector $\mathbf{a}_{1}^{\mathbf{r}}$ and introduces a constraint between $u_{1}, u_{3}$ and $\theta$.

The coordinates of the material point $M$ in the local frame $\left(G, \mathbf{e}_{\mathbf{2}}^{\mathbf{r}}, \mathbf{e}_{\mathbf{3}}^{\mathbf{r}}\right)$ are given by $y\left(s_{1}, s_{2}, t\right)$ and $z\left(s_{1}, s_{2}, t\right)$. Thus assumption (iii), which is equivalent to introducing an elastica to describe the kinematics of the cross-section curve, leads to the following relation:

$$
\left\|\mathbf{G M}_{, 2}\left(s_{1}, s_{2}, t\right)\right\|-\left\|\mathbf{G}_{\mathbf{0}} \mathbf{M}_{\mathbf{0}, 2}\left(s_{2}\right)\right\|=\left(y_{, 2}\left(s_{1}, s_{2}, t\right)\right)^{2}+\left(z_{, 2}\left(s_{1}, s_{2}, t\right)\right)^{2}-1=0
$$

where $X_{, i}$ stands for the partial derivative of $X$ with respect to $s_{i}$. By introducing the angle $\beta\left(s_{1}, s_{2}, t\right)$ between the tangent to the cross-section curve and the vector $\mathbf{e}_{2}^{\mathbf{r}}$ (see Figure 2), we get:

$$
\left\{\begin{array}{l}
y_{, 2}\left(s_{1}, s_{2}, t\right)=\cos \beta\left(s_{1}, s_{2}, t\right) \\
z_{, 2}\left(s_{1}, s_{2}, t\right)=\sin \beta\left(s_{1}, s_{2}, t\right)
\end{array}\right.
$$

When making assumption (iii), we suppose that the most important effect governing the changes in the cross-section shape is the adjustment of the overall bending inertia of the rod in order to minimize its elastic energy: the flattening of the cross-section concentrates the bending deformation and makes the formation of localized folds possible. In doing so we suppose that transverse strains can be neglected when calculating the tape spring bending inertia and that the inextensibility assumption is enough to describe the overall shape of the cross-section curve. It is thus possible to use only one kinematic parameter $\beta\left(s_{1}, s_{2}, t\right)$ to describe the behavior of the cross-section for large displacements and large rotations. This way of parametrizing the cross-section curve is clearly inspired by the Elastica theory ${ }^{3,5}$.

Moreover, we suppose that the cross-section curve stays circular with a curvature radius function of $s_{1}$ only, and we introduce the opening angle of the cross-section curve $\beta^{e}\left(s_{1}, t\right)=\beta\left(s_{1}, s_{2}=a / 2, t\right)$. Thus the angle between $\mathbf{e}_{2}$ and the tangent to the cross-section curve can be written as a function of $s_{2}$ :

$$
\beta\left(s_{1}, s_{2}, t\right)=\frac{2 s_{2}}{a} \beta^{e}\left(s_{1}, t\right)
$$

Finally, the expression of $\beta^{e}\left(s_{1}, t\right)$ makes it possible to go back from $y_{, 2}$ and $z_{, 2}$ to the local coordinates $y$ and $z$ :

$$
\left\{\begin{array}{l}
y\left(s_{1}, s_{2}, t\right)=\int_{0}^{s_{2}} \cos \beta\left(s_{1}, \xi, t\right) \mathrm{d} \xi=\frac{a}{2 \beta^{e}\left(s_{1}, t\right)} \sin \left(\frac{2 s_{2}}{a} \beta^{e}\left(s_{1}, t\right)\right) \\
z\left(s_{1}, s_{2}, t\right)=\int_{0}^{s_{2}} \sin \beta\left(s_{1}, \xi, t\right) \mathrm{d} \xi=\frac{a}{2 \beta^{e}\left(s_{1}, t\right)}\left(1-\cos \left(\frac{2 s_{2}}{a} \beta^{e}\left(s_{1}, t\right)\right)\right)
\end{array}\right.
$$

Thus the tape spring kinematics is totally described by four kinematic parameters attached to the rod line and functions of $s_{1}$ and $t$ only:

(i) the translations $u_{1}$ and $u_{3}$ of the reference point of the cross-section,

(ii) the rotation $\theta$ of the cross-section plane around $\mathbf{e}_{2}$,

(iii) and the angle $\beta^{e}$ characterizing the opening of the cross-section.

The approach presented herein for relatively simple cross-section shape and kinematics may be generalized to more complex shapes or kinematics by choosing an appropriate discretization (e.g. Ritz, FE...) of the angle $\beta\left(s_{1}, s_{2}, t\right)$ with respect to the transverse coordinate $s_{2}$ and by adding some kinematic parameters.

\section{II.B. Strains measures and strain energy}

At the beginning the tape spring is regarded as a thin shell. The strain energy is thus calculated using the membrane and bending strains defined respectively by the Green-Lagrange tensor and by the difference between the initial and actual curvature tensors of the shell. All tensors are expressed thanks to the kinematic parameters $u_{1}, u_{3}, \theta$ and $\beta^{e}$. 
Since the shell thickness $h$ is very small compared to the length of the cross-section curve $a$, local and global buckling will prevent large strains to occur, making the assumption of small membrane strains possible. Moreover only the tensile membrane strain $e_{11}$ is needed due to further assumptions that will be exposed later on. The simplified expressions for the membrane strains and bending curvatures are then:

$$
\begin{gathered}
\qquad\left\{\begin{array}{l}
e_{11}=e^{r}+z k^{r}+e^{s} \\
k_{11}=-k^{r} \cos \beta+k_{11}^{s} \\
k_{22}=k_{22}^{s} \\
k_{12}=k_{12}^{s}
\end{array}\right. \\
\text { where }\left\{\begin{array} { l } 
{ e ^ { r } = u _ { 1 , 1 } + \frac { 1 } { 2 } ( u _ { 1 , 1 } ^ { 2 } + u _ { 3 , 1 } ^ { 2 } ) } \\
{ k ^ { r } = \theta _ { , 1 } }
\end{array} \text { and } \left\{\begin{array}{l}
e^{s}=\frac{1}{2}\left(y_{, 1}^{2}+z_{, 1}^{2}\right) \\
k_{11}^{s}=z_{, 11} \cos \beta-y_{, 11} \sin \beta \\
k_{22}^{s}=\beta_{, 2}-\beta_{0,2} \\
k_{12}^{s}=\beta_{, 1}
\end{array}\right.\right.
\end{gathered}
$$

Expression (7) shows strains induced by the global rod kinematics (usual tensile strain $e^{r}$ and bending curvature $k^{r}$ for large displacements and large rotations) and strains induced by the cross-section evolution $\left(e^{s}\right.$ and $k_{\alpha \beta}^{s}$ only depending on the angle $\beta$ ) which constitute the originality of the model.

The strain energy of an elastic shell is set to its usual expression:

$$
U_{e}\left(u_{1}, u_{3}, \theta, \beta\right)=\int_{0}^{L} \int_{-a / 2}^{a / 2} \frac{1}{2}\left(e_{\alpha \beta} N_{\alpha \beta}+k_{\alpha \beta} M_{\alpha \beta}\right) d s_{2} d s_{1} .
$$

where $N_{\alpha \beta}$ and $M_{\alpha \beta}$ are respectively the membrane stresses and bending moments. Since the shell width $a$ is small compared to the tape spring length $L$, we suppose that $N_{22}=N_{12}=0$ according to classical beam theory assumptions. Moreover the shell is considered elastic and orthotropic without any membrane-bending coupling. Thus the constitutive equations of the shell are written:

$$
N_{11}=A e_{11} \quad M_{11}=D_{1} k_{11}+D_{3} k_{22} \quad M_{22}=D_{3} k_{11}+D_{2} k_{22} \quad M_{12}=D_{4}\left(2 k_{12}\right)
$$

with $A, D_{1}, D_{2}, D_{3}$ and $D_{4}$ the elastic constants of the shell.

Finally, the strain energy can be split into three parts:

$$
\begin{gathered}
U_{e}(t)=U_{e}^{r}(t)+U_{e}^{s}(t)+U_{e}^{r s}(t) \\
\left\{\begin{array}{l}
U_{e}^{r}(t)=\int_{0}^{L} \frac{1}{2}\left(A a\left(e^{r}\right)^{2}+\left(A \overline{z^{2}}+D_{1} \overline{\cos ^{2} \beta}\right)\left(k^{r}\right)^{2}+2 A \bar{z} e^{r} k^{r}\right) \mathrm{d} s_{1} \\
U_{e}^{s}(t)=\int_{0}^{L} \frac{1}{2}\left(A \overline{\left(e^{s}\right)^{2}}+D_{1} \overline{\left(k_{11}^{s}\right)^{2}}+D_{2} \overline{\left(k_{22}^{s}\right)^{2}}+2 D_{3} \overline{k_{11}^{s} k_{22}^{s}}+4 D_{4} \overline{\left(k_{12}^{s}\right)^{2}}\right) \mathrm{d} s_{1} \\
U_{e}^{r s}(t)=\int_{0}^{L}\left(A e^{r} \overline{e^{s}}+A k^{r} \overline{z e^{s}}-k^{r}\left(D_{1} \overline{\cos \beta k_{11}^{s}}+D_{3} \overline{\cos \beta k_{22}^{s}}\right)\right) \mathrm{d} s_{1}
\end{array}\right.
\end{gathered}
$$

where the overline denotes an integration with respect to $s_{2}: \bar{X}\left(s_{1}, t\right)=\int_{-a / 2}^{a / 2} X\left(s_{1}, s_{2}, t\right) \mathrm{d} s_{2}$.

For the model presented in this section all these integrals can be calculated analytically, providing an explicit expression of the strain energy density depending on the kinematic parameters and geometric characteristics of the tape spring. The first term $U_{e}^{r}$ corresponds to the classical strain energy of a rod with a coupling between axial stretching and bending which appears because the rod line does not pass through the cross-section centroid. The second term $U_{e}^{s}$ only depends on the variable $\beta$ and represents the strain energy due to the variation of the cross-section shape, independently from the overall rod behavior. The last term $U_{e}^{r s}$ introduces a coupling between the overall rod behavior and the variation of the cross-section shape. 


\section{II.C. Kinetic energy}

Starting from the kinetic energy of the initial shell model and neglecting the rotation inertia, we introduce the chosen kinematics and obtain the following expression for the kinetic energy:

$$
\begin{gathered}
T(t)=T^{r}(t)+T^{s}(t)+T^{r s}(t) \\
\text { with }\left\{\begin{array}{l}
T^{r}(t)=\int_{0}^{L} \frac{1}{2}\left(a \rho^{s}\left(\dot{u}_{1}^{2}+\dot{u}_{3}^{2}\right)+\rho^{s} \overline{z^{2}} \dot{\theta}^{2}\right) \mathrm{d} s_{1} \\
T^{s}(t)=\int_{0}^{L} \frac{1}{2} \rho^{s}\left(\overline{\dot{y}^{2}+\dot{z}^{2}}\right) \mathrm{d} s_{1} \\
T^{r s}(t)=\int_{0}^{L} \rho^{s}\left(-\dot{u}_{1}(\widehat{\overline{z \sin \theta}})+\dot{u}_{3}(\widehat{\overline{z \cos \theta}})\right) \mathrm{d} s_{1}
\end{array}\right.
\end{gathered}
$$

where the notation $\dot{X}$ stands for the time derivative of $X$.

\section{Numerical results}

\section{III.A. Numerical Implementation}

For the numerical simulations we use the FE software COMSOL to solve the weak formulation of the elastodynamic problem obtained from our one-dimensional model applying the Hamilton principle. Indeed COMSOL offers the possibility to introduce directly the expressions for the elastic and kinetic energies and is able to proceed to an analytical differentiation of these expressions. Only the kinetic energy $T$ requires an explicit calculus of variations because an integration by parts with respect to time is needed. A Lagrange multiplier (fifth degree of freedom) is used to constrain the translations $u_{1}$ and $u_{3}$ and the rotation $\theta$. Finally, a finite element discretization of the rod line is done before solving this constraint problem for the stationary points of the Hamiltonian.

\begin{tabular}{|c|c|c|c|c|c|c|}
\hline $\begin{array}{c}\text { Length } \\
L\end{array}$ & $\begin{array}{c}\text { Width } \\
a\end{array}$ & $\begin{array}{c}\text { Thickness } \\
h\end{array}$ & $\begin{array}{c}\text { Initial } \\
\text { angle } \beta_{0}^{e}\end{array}$ & $\begin{array}{c}\text { Young } \\
\text { modulus } E\end{array}$ & $\begin{array}{c}\text { Poisson } \\
\text { ratio } \nu\end{array}$ & $\begin{array}{c}\text { Density } \\
\rho\end{array}$ \\
\hline $1170 \mathrm{~mm}$ & $60 \mathrm{~mm}$ & $0.15 \mathrm{~mm}$ & $0.6 \mathrm{rad}$ & $210000 \mathrm{MPa}$ & 0.3 & $7800 \mathrm{~kg} \cdot \mathrm{m}^{-3}$ \\
\hline
\end{tabular}

Table 1. Tape spring properties

The results presented hereafter demonstrate the ability of the model to account for complex scenarios of folding, coiling and deployment in dynamics, showing very fast phenomenons like pendulum or inertia effects. The geometric and material properties of the studied tape spring are presented in Table 1. In the following figures, the 3D deformed shapes have been rebuilt from the one-dimensional rod model solution and the kinematics exposed in Section II. Other examples may be found in the following references ${ }^{6,7}$.

\section{III.B. Static test - Splitting of a fold into two}

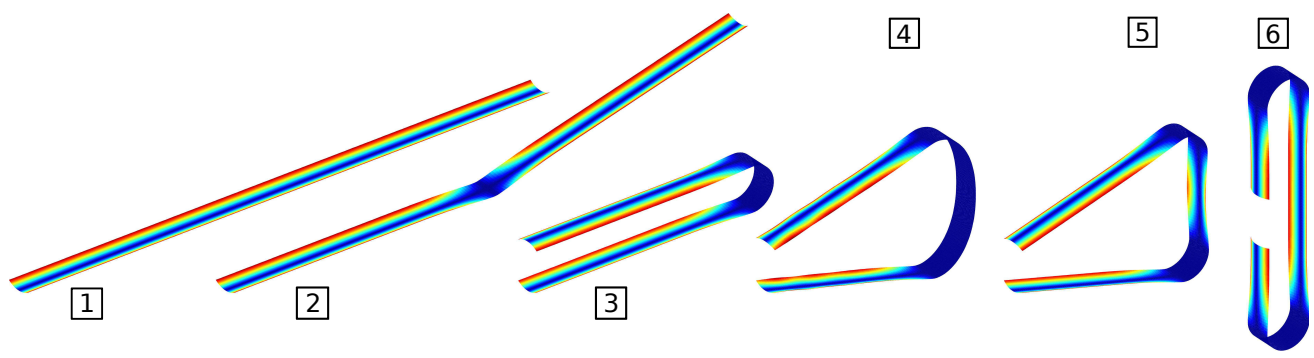

Figure 3. Folding of a tape spring followed by the splitting of the fold into two. 
This first example (see Figure 3) illustrates the capacity of the model to account for large rotations as well as the creation of a fold and its parting into two. The left end of the tape spring being clamped, a first fold is created by imposing a flattening moment (associated to the kinematic parameter $\beta^{e}$ ) on the central cross-section (see deformed shape 2 ). A rotation $\theta$ of the cross-section is then applied at the other end until both arms of the tape are collinear, the flattening moment being released at the same time (see deformed shape 3 ). From Figure 4 we notice several characteristics of a tape spring folding area :

(i) cross-sections are flat in the fold area (see Figure 4 (a)),

(ii) and the fold radius is constant, equal to the slope of the curve of $\theta$ function of $s_{1}$ (see Figure 4 (b)) as well as equal to the transverse curvature radius of the undeformed cross-section.

Displacements $u_{1}$ and $u_{3}$ are then fixed at both ends and opposite increments of rotation are imposed leading to the splitting of the fold into two new folds (see deformed shapes 44 and 5 ) which move along the tape until both arms become vertical (see deformed shape 6). During the first spacing step (deformed shapes 4 and 5 ) the flat area (i.e. the fold region) spreads along the tape and localized axial compression occurs along the straight parts of the tape spring because the global buckling induces axial compression along the tape longitudinal edges. This leads to a buckling mode in which the cross-section shape oscillates to relieve the compression and results in oscillations (see Figure 4 (a)) of the value of the opening angle $\beta^{e}$ of the cross-section curve.
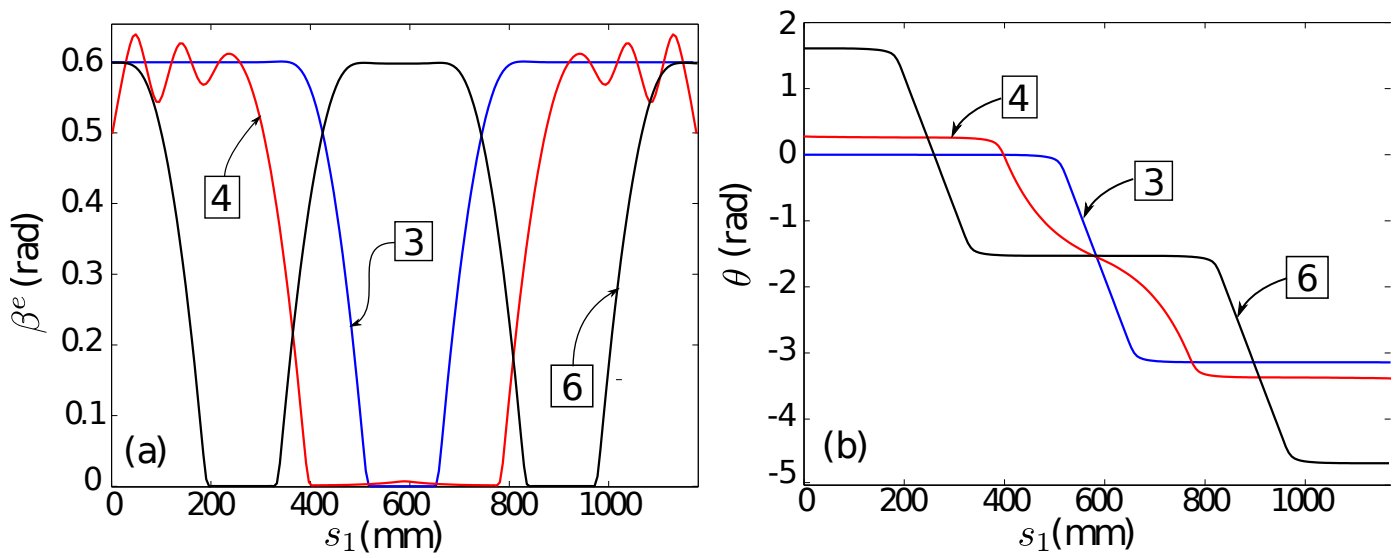

Figure 4. Evolution of $\beta^{e}$ and $\theta$ during the loading.

The snapthrough that happens between states 4 and 5 makes the compressive stresses disappear and leads to the creation of a second fold. In Figure 4 (a), we observe the beginning of the snapthrough with a small reclosing of the cross-section at the middle of the tape (see curve 4 ) and we see that the region between the two folds regains its initial transverse curvature (see curves 3 and 6 ). Looking at Figure 4 (b) we also notice that the flat area of both folds have the same length and folding radius. Moreover this coiling radius is almost equal to the transverse curvature radius of the undeformed tape spring, which is in perfect agreement with the geometrical properties characterizing the fold area exposed by Seffen \& Pellegrino ${ }^{12}$.

\section{III.C. Dynamic test - Uncoiling of a partially coiled tape spring}

This second example shows one of the uncoiling modes of a tape spring as described in ${ }^{12}$. In the studied case (see Figure 5), the tape is partially coiled with a coiling radius equal to the curvature radius of the cross-section in the undeformed configuration. When the end cross-section of the straight part of the tape is released, the coiled part keeps a constant coiling radius during the whole deployment phase. This would not be the case if the coiling radius was not equal to the curvature radius, indeed the coiling radius would have increased during the deployment, making the uncoiling of the tape spring less regular. 

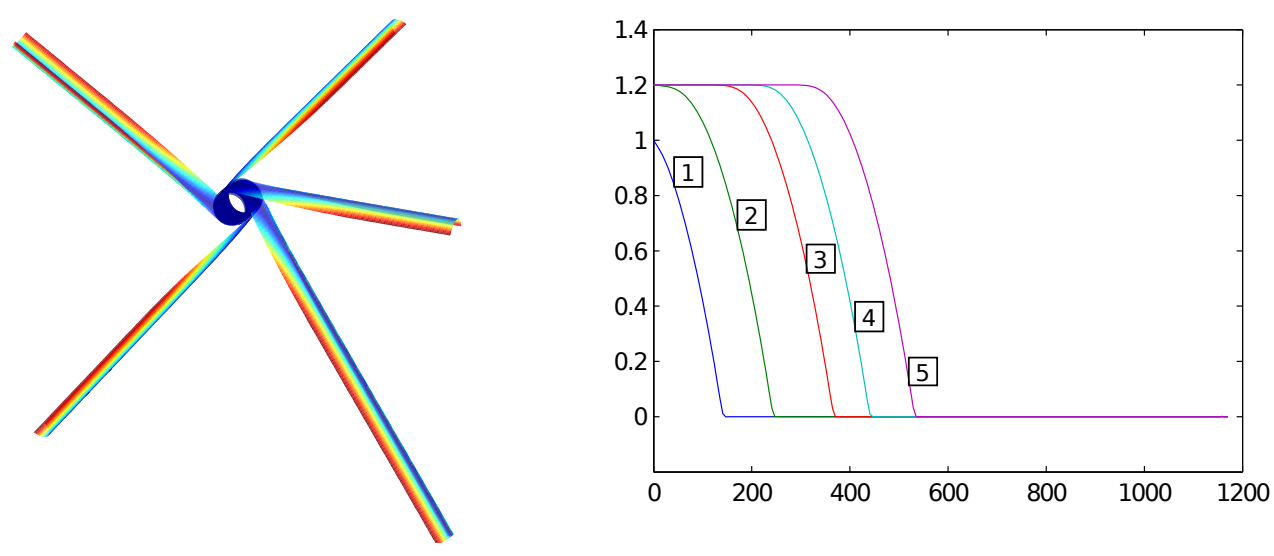

Figure 5. Uncoiling of a partially coiled tape spring and evolution of $\beta^{e}$ during the deployment.

\section{III.D. Experimental comparison - Deployment of a tape spring with a folded part}

In this last example we compare our numerical results to experimental ones obtained by Seffen \& Pellegrino in $1999^{12}$. The tape, subjected to gravity, is vertical and clamped at the bottom end whereas the other end is folded on half the total length (see Figure 6). The folded end is then released instantaneously without initial speed. During the first step of deployment, the fold moves down along the tape as the free end straightens up progressively. The clamped boundary condition prevents the bottom cross-section from flattening, causing the fold to rebound when it arrives at the bottom of the tape. As the fold moves up, the free arm of the tape goes through the vertical position and the inertia effects cause the formation of a new fold of opposite curvature in the fold area before its complete disappearance. This new fold is also going to move along the tape before the tape recovers its undeformed shape. We also observe compressive stresses in both arms of the tape spring during the second step of deployment.
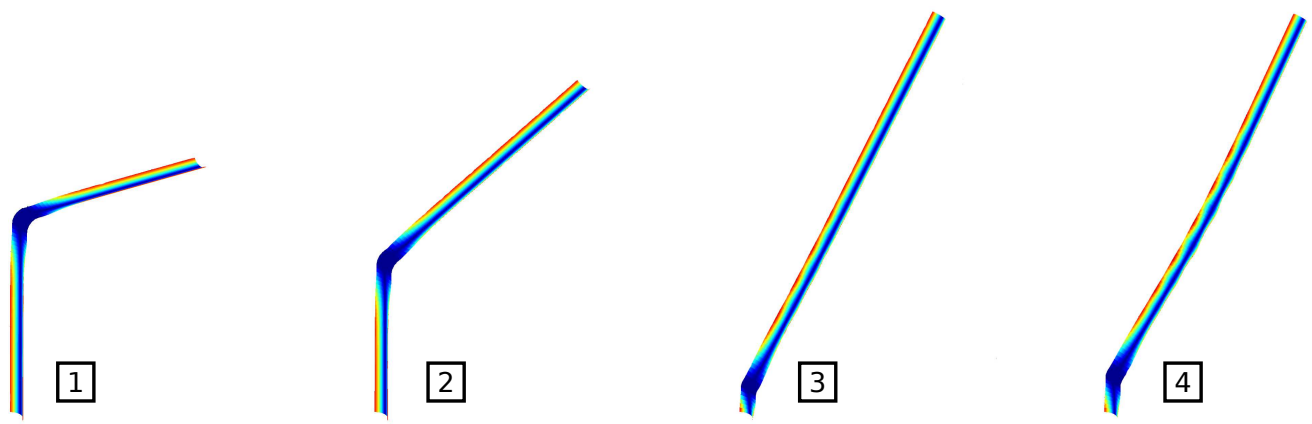

Figure 6. Deployment of a tape spring with a folded part.

This test was first simulated without any damping (see Figure 7 (a)). In this case, our model gives correct results in the beginning but becomes less accurate after the first rebound of the fold against the clamped boundary condition (see deformed shapes 3 and 4 ). By adding a weak damping to our model, the results obtained for the same test are now accurate until the second rebound of the fold (see Figure 7 (b)). Furthermore, after the $2^{\text {nd }}$ rebound three-dimensional effects occurs in the experimental case that cannot be taken into account with our planar model, thus it is not pertinent to compare our results to the experimental case after this $2^{\text {nd }}$ rebound. Since the damping in the experimental case seems to come mainly from the rebound of the fold on the clamped boundary condition, the damping in our model has been applied to the kinematic variable $\beta^{e}$ which is the most affected by the rebound. 

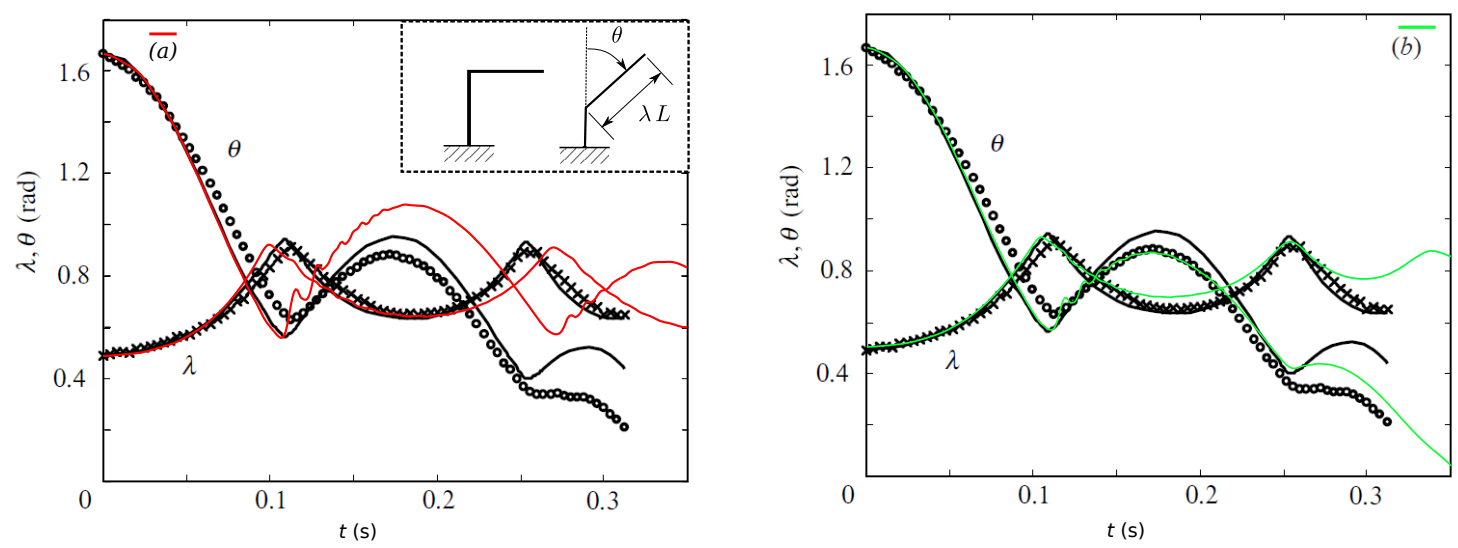

Figure 7. Evolution of $\theta$ and $\lambda$ during the deployment. Dots and crosses refer to experiment, black lines refer to Seffen \& Pellegrino discrete model ${ }^{12}$, red and green lines refer to our model ${ }^{7}$.

\section{Conclusion}

In this work a rod model with highly deformable cross-section suitable for thin-walled and curved crosssections has been exposed. This model accounts for large displacements, large rotations and dynamics. The equations and results submitted in this paper are for the limiting case of symmetric (i.e. in-plane) displacements but the case of non-symmetric displacements including out-of-plane bending and torsion is currently being studied. In both cases the approach used is the same. Starting from a classical shell model, an elastica kinematics is introduced to account for the changes of the cross-section shape. Moreover beamlike assumptions are made, e.g. by neglecting the transverse membrane stresses when compared to the axial membrane stresses. The elastic and kinetic energies are then derived and the model is implemented in the FE software COMSOL, allowing to use the Hamilton principle to solve the elastodynamic problem.

The one-dimensional model with only four degrees of freedom (in-plane displacements case) has been used to model several sequences of folding, coiling and deployment of a tape spring. It has proved its ability to account for several phenomena such as the sudden creation of a fold, the splitting of a fold into two, the inertia or pendulum effects... Its generalization to the out-of-plane case should allow the modeling of the deployment and the stability of more complex structures, made for example of an assembly of tape springs.

\section{Acknowledgments}

This work was supported by doctoral grants from Thales Alenia Space, the region of Provence-Alpes-Côte d'Azur and the CNRS (Scientific Research National Center). The support is gratefully acknowledged.

\section{References}

${ }^{1}$ B. Audoly, Y. Pomeau, Elasticity and Geometry: From hair curls to the nonlinear response of shells, Oxford University Press, 2010.

${ }^{2}$ B. Budiansky, Theory of buckling and post-buckling behaviour of elastic structures, Advances in Applied Mechanics 14 1-65, 1974.

${ }^{3}$ E. Euler, Methodus inveniendi lineas curvas maximi minimive proprietate gaudentes, 1744 .

${ }^{4}$ R. Gonçalves, M. Ritto-Corrêa, D. Camotim, A large displacement and finite rotation thin-walled beam formulation including cross-section deformation, Comput. Meths. Appl. Mech. Engrg. 199 1627-1643, 2010.

${ }^{5}$ V.G.A. Goss, The History of the Planar Elastica: Insights into Mechanics and Scientific Method, Science \& Education, 18 1057-1082, 2009.

${ }^{6} \mathrm{~F}$. Guinot, Déploiement régulé de structures spatiales : vers un modèle unidimensionnel de mètre ruban composite, Doctoral Thesis, Université de Provence, Marseille, 2011.

${ }^{7}$ F. Guinot, S. Bourgeois, B. Cochelin, L. Blanchard, A planar rod model with highly flexible thin-walled cross-sections. Application to the folding of tape springs., International Journal of Solids and Structures 49 73-86, 2012.

${ }^{8}$ S. Hoffait, O. Bruls, D. Granville, F. Cugnon, G. Kerschen, Dynamic analysis of the self-locking phenomenon in tape-spring hinges, Acta Astronautica 66 1125-1132, 2009. 
${ }^{9}$ Q.S. Nguyen, Stability and nonlinear solid mechanics, Wiley, Chichester.

${ }^{10}$ P.M. Pimenta, E.M.B. Campello, A fully nonlinear multi-parameter rod model incorporating general cross-sectional inplane changes and out-of-plane warping, Latin American Journal of Solids and Structures 1 119-140, 2003.

${ }^{11}$ M. Santer, S. Pellegrino, Compliant multistable structural elements, International Journal of Solids and Structures 45 6190-6204, 2008.

${ }^{12}$ K. Seffen, S. Pellegrino, Deployment dynamics of tape springs, Proceedings of the Royal Society A 455 1003-1048, 1999.

${ }^{13}$ K. Seffen, Z. You, S. Pellegrino, Folding and deployment of curved tape springs, International Journal of Mechanical Sciences 42 2055-2073, 2000.

${ }^{14}$ N.A.F Senan, O.M. O'Reilly, T.N. Tresierras, Modeling the growth and branching of plants: a simple rod based model, Journal of the Mechanics and Physics of Solids 56 (10) 3021-3036, 2008.

${ }^{15}$ E. Sarostin, G. van der Heijden, Cascade unlooping of a low-pitch helical spring under tension, Journal of the Mechanics and Physics of Solids 57 959-969, 2009.

${ }^{16}$ S.P. Timoshenko, J.M. Gere, Theory of elastic stability, Mc Graw-Hill, New-York, 1961.

${ }^{17}$ B.Z. Vlassov, Pièces longues en voiles minces, Eyrolles, Paris, 1962.

${ }^{18}$ S.J.I. Walker, G.S. Aglietti, A study of tape spring fold curvature for space deployable structures, Proc. of the Institution of Mech. Engrs., Part G (J. of Aerospace Engrg.), G3 221 313-325, 2007.

${ }^{19}$ M. Zivkovic, M. Kojic, R. Slavkovic, N. Grujovic, A general beam finite element with deformable cross-section, Comput. Meths. Appl. Mech. Engrg. 190 2651-2680, 2001. 